\title{
Source structure: an essential piece of information for generating the next ICRF
}

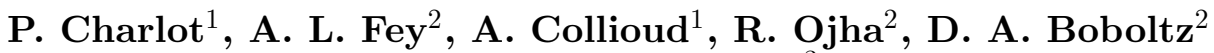 \\ and J. I. B. Camargo ${ }^{3}$ \\ ${ }^{1}$ Laboratoire d'Astrophysique de Bordeaux, Université Bordeaux 1, CNRS \\ BP 89, 33270 Floirac, France \\ email: charlot@obs.u-bordeaux1.fr \\ ${ }^{2}$ U.S. Naval Observatory \\ 3450 Massachusetts Ave., NW, Washington, DC 20392-5420, USA \\ ${ }^{3}$ Observatorio do Valongo, Universidade Federal do Rio de Janeiro \\ Ladeira do Pedro Antônio, 43, Rio de Janeiro, RJ, Brazil
}

\begin{abstract}
The intrinsic radio structure of the extragalactic sources is one of the limiting factors in defining the International Celestial Reference Frame (ICRF). This paper reports about the ongoing work to monitor the structural evolution of the ICRF sources by using the Very Long Baseline Array and other VLBI telescopes around the world. Based on more than 5000 VLBI images produced from such observations, we have assessed the astrometric suitability of $80 \%$ of the ICRF sources. The number of VLBI images for a given source varies from 1 for the least-observed sources to more than 20 for the intensively-observed sources. Overall, we identify a subset of 194 sources that are highly compact at any of the available epochs and which are prime candidates for the realization of the next ICRF with the highest accuracy.
\end{abstract}

Keywords. astrometry, reference systems, quasars: general

\section{Introduction}

The International Celestial Reference Frame (ICRF), which has been the official IAU reference frame in use since 1 January 1998, is currently based on the VLBI positions of 717 extragalactic radio sources. Of these, 608 sources are from the original ICRF built in 1995, with a categorization that comprises 212 well-observed defining sources (which served to set the axes of the frame), 294 less-observed candidate sources, and 102 other sources showing coordinate instabilities (Ma et al. 1998). The accuracy of the individual ICRF source positions has a floor of $250 \mu \mathrm{as}$, while the axes of the frame are stable to about $20 \mu$ as. Since then the positions have been improved for the non-defining sources and the frame has been extended by 109 new sources in ICRF-Ext.1 and ICRF-Ext.2 using additional data acquired in the period 1995-2002 (Fey et al. 2004).

At the IAU XXVI ${ }^{\text {th }}$ General Assembly in Prague (August 2006), the community decided to engage in realization of the successor to ICRF, to be presented at the next IAU General Assembly in 2009. The motivation for generating this new celestial frame is to benefit from recent improvements in VLBI modeling (e.g. for the troposphere) and to take advantage of the wealth of VLBI data that have been acquired since the time the ICRF was established. A major issue to be addressed in this new realization is the revision of source categorization, in particular the choice of the defining sources. Such a revision is necessary because some of the original ICRF defining sources are found to have extended structures (Fey \& Charlot 2000) or position instabilities (e.g. MacMillan 2006), and are therefore inadequate for defining the celestial frame with the highest accuracy. 

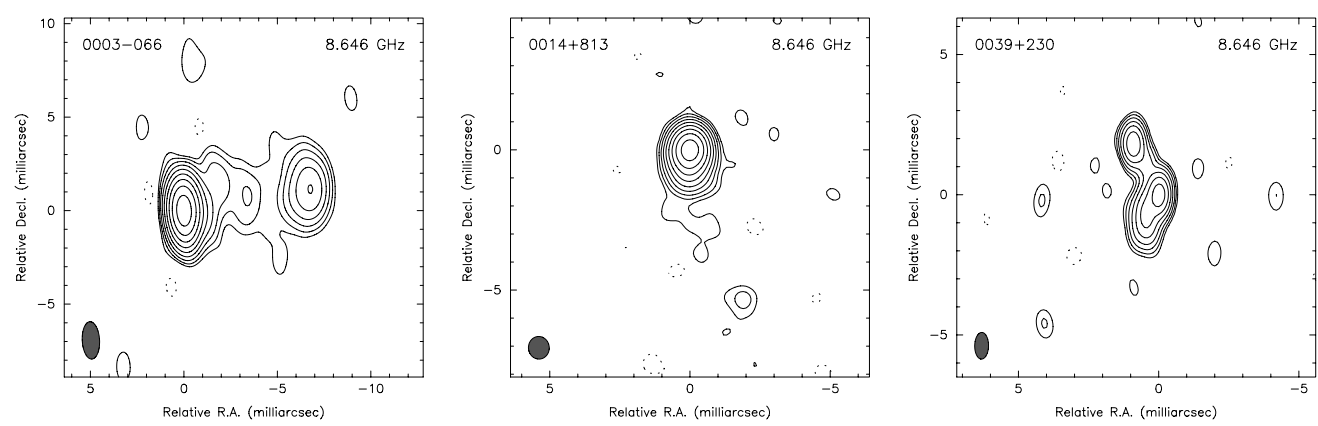

Figure 1. VLBI images at $\mathrm{X}$ band $(8 \mathrm{GHz})$ for three ICRF sources $(0003-066,0014+813$, $0039+230$ ) as derived from the data of a RDV session conducted on 2003 December 17. The three sources were selected randomly according to increasing right ascension starting at $\mathrm{RA}=00 \mathrm{~h}$.

The selection criteria that are considered by the working group in charge of the next ICRF realization are based either on source structure information (VLBI images), to evaluate the compactness and astrometric suitability of the sources, or on time series of source coordinates, to assess the source position stability. In this paper, we discuss only the former. There are now more than 5000 VLBI images available to evaluate source quality, whereas these were less than a hundred at the time the ICRF was built, thereby permitting considerable progress. In Sect. 2, we present the observational data and analysis method used in this work. Our results are discussed in Sect. 3 with emphasis on statistics of source quality for each ICRF source category and for the overall ICRF. We also draw prospects for the further improvements in assessing the source quality before the next ICRF is generated.

\section{Observational data and analysis method}

The VLBI maps used in our analysis were produced from a total of 38 VLBI sessions conducted between 1994 and 2007, which were imaged either at USNO or at Bordeaux Observatory. These comprise:

- 8 dedicated dual-frequency $(8 \mathrm{GHz} / 2 \mathrm{GHz})$ imaging sessions conducted with the Very Long Baseline Array (VLBA) between July 1994 and January 1997 (Fey et al. 1996, Fey \& Charlot 1997, Fey \& Charlot 2000);

- 23 dual-frequency $(8 \mathrm{GHz} / 2 \mathrm{GHz}$ ) Research \& Development VLBA (RDV) sessions conducted between January 1997 and June 2007; these sessions include the 10 VLBA stations and up to 10 additional geodetic telescopes;

- 5 dedicated southern-hemisphere $8 \mathrm{GHz}$ imaging sessions conducted between July 2002 and April 2004 with the Australian Long baseline Array, augmented by radio telescopes in South-Africa, Hawaii, and Japan (Ojha et al. 2004, Ojha et al. 2005);

- 2 VLBA sessions at $2 \mathrm{GHz} / 8 \mathrm{GHz} / 24 \mathrm{GHz}$ and $8 \mathrm{GHz} / 24 \mathrm{GHz}$ conducted in February 2004 and August 2005 as part of a project to extend the ICRF to higher frequencies (Lanyi et al. 2007).

Altogether, this represents a total of 2697 maps at $\mathrm{X}$ band $(8 \mathrm{GHz})$ from $577 \mathrm{ICRF}$ sources and 2388 maps at $\mathrm{S}$ band $(2 \mathrm{GHz})$ from $492 \mathrm{ICRF}$ sources. Less sources have been imaged at $\mathrm{S}$ band because the southern-hemisphere sessions did not observe at this band. There are up to 28 VLBI epochs available for the most intensively-observed source, whereas only one epoch is available for the least-observed sources. A sample of $\mathrm{X}$-band VLBI images for three ICRF sources, as derived from the data of a RDV session conducted in December 2003, is shown in Fig. 1. From these images, only one source 
$(0014+813)$ is relatively compact, while the other two (0003-066 and $0039+230)$ show extended structures. This fraction of compact sources is consistent with that found for the whole ICRF, as discussed in Sect. 3 .

Based on the VLBI images, we derived the expected effects of intrinsic source structure on the VLBI delay astrometric quantities, following the algorithm of Charlot (1990). We then used the "structure index" indicator to define the astrometric source quality, as devised by Fey \& Charlot (1997). Structure index values of 1 and 2 point to excellent and good astrometric suitability, respectively, while values of 3 and 4 indicate a poor suitability. A given source may have differing structure indices at $\mathrm{X}$ band and $\mathrm{S}$ band, depending on properties of the brightness distribution at each band. The structure index may also vary with time because of possible temporal evolution of the brightness distribution.

For each source, we obtained a series of structure index at each band according to the number of available VLBI images (e.g., Charlot et al. (2006)). Adopting a conservative approach, we chose the maximum value of structure index as the source quality indicator when multi-epoch structure indices are available. In other words, if a source shows a structure index value of 3 or 4 at one or more epochs, it should be regarded as unsuitable for highly-accurate astrometry even though it has the structure index values of 1 or 2 at some other epochs. This multi-epoch structure index value is the criterion used for drawing statistics on the source quality.

\section{Results}

As noted above, we have obtained structure indices for 577 sources in $\mathrm{X}$ band and 492 sources in S band (representing $80 \%$ and $69 \%$ of the current 717 sources of the ICRF, respectively). The X-band structure index distribution (Fig. 2) shows that 197 sources (or $34 \%$ of all sources) are astrometrically-suitable at this frequency according to our criterion (structure index is either 1 or 2 ). In $\mathrm{S}$ band, $86 \%$ of the sources have a structure index either 1 or 2 (Fig. 2). This indicates that the contribution of the Sband structure to the dual-frequency $(\mathrm{S} / \mathrm{X}$ ) calibrated delay is usually smaller compared to the contribution by X-band structure, as already noted in Fey \& Charlot (1997). Comparing the $\mathrm{X}$ - and S-band structure indices for each source shows that, with three exceptions, all sources that have a S-band structure index of either 3 or 4 have also a Xband structure index of 3 or 4 . Based on the S-band structure index, we thus exclude only 3 additional sources, which leaves a total of 194 ICRF sources astrometrically-suitable at both frequencies.

In Fig. 3, the X-band structure index distribution is compared for each ICRF source category. As expected, the distribution is somewhat better for the defining sources than for the candidate and "other" sources. However, only about $40 \%$ of the ICRF defining sources have a structure index value of either 1 or 2 . The fraction of suitable sources drops down to $32 \%$ for the candidate sources and $22 \%$ for the "other" sources, while it is $48 \%$ for the "new" sources. Overall, these results confirm that revision of source categories is mandatory for the next ICRF.
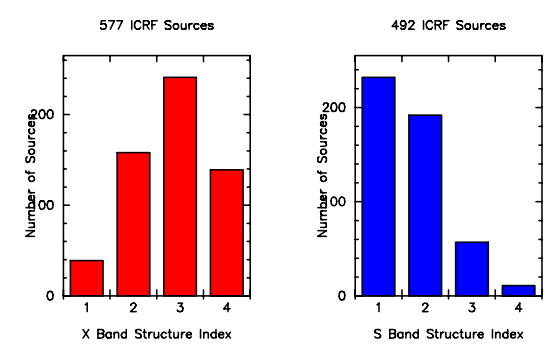

Figure 2. The structure index distribution at $\mathrm{X}$ band $(8 \mathrm{GHz})$ and $\mathrm{S}$ band $(2 \mathrm{GHz})$ for all ICRF sources that have a structure index available at these frequencies. 

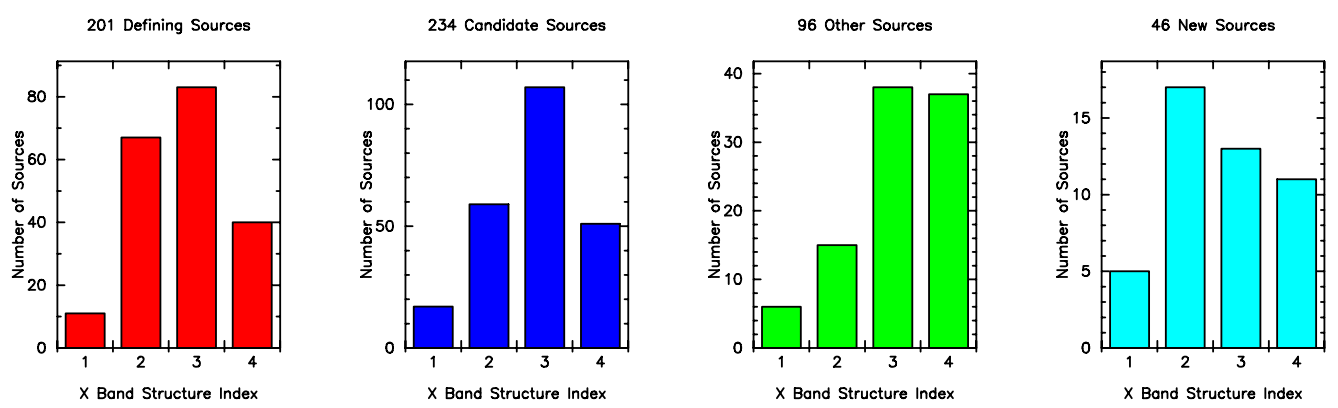

Figure 3. Distribution of the X-band ( $8 \mathrm{GHz}$ ) structure indices in each ICRF source category (defining, candidate, "other", "new"). The 577 ICRF sources with currently available structure indices are included.

\section{Conclusions}

We have evaluated the astrometric suitability of $80 \%$ of the sources in the ICRF based on multi-epoch VLBI maps of their structures. From this analysis, a sample of 194 astrometrically-suitable ICRF sources that have compact or very compact structures according to our "structure index" indicator has been identified. It is anticipated that the remaining $20 \%$ of ICRF sources for which the astrometric suitability has not been assessed (mostly in the southern sky) will be imaged in the near future through further VLBI observing programs in the southern hemisphere. The astrometric suitability of the sources already imaged, and discussed in this paper, will also be refined as new VLBI sessions are processed and maps become available. This information will be essential for selecting the proper defining sources and generating the next ICRF by 2009 .

\section{References}

Charlot P. 1990, AJ 99, 1309

Charlot, P., Fey, A. L., Ojha, R., \& Boboltz, D. A. 2006, in: International VLBI Service for Geodesy and Astrometry 2006 General Meeting Proceedings, Eds. D. Behrend and K. D. Baver, NASA/CP-2006-214140, p. 321.

Fey, A. L., Clegg, A. W., \& Fomalont, E. B. 1996, ApJS 105, 299

Fey, A. L. \& Charlot, P. 1997, ApJS 111, 95

Fey, A. L. \& Charlot, P. 2000, ApJS 128, 17

Fey, A. L., Ma, C., Arias, E. F., Charlot, P., Feissel-Vernier, M., Gontier, A.-M., Jacobs, C. S., Li, J., \& MacMillan, D. S. 2004, AJ 127, 3587

Lanyi, G. E., Jacobs, C. S., Naudet, C. J., Zhang, L. D., Boboltz, D. A., Fey, A. L., Charlot, P., Fomalont, E. B., Geldzahler, B., Gordon, D., Ma, C., Romney, J. E., \& Sovers, O. J. 2007, $A J$ (in preparation)

Ma, C., Arias, E. F., Eubanks, T. M., Fey, A. L., Gontier, A.-M., Jacobs, C. S., Sovers, O. J., Archinal, B. A., \& Charlot, P. 1998, AJ 116, 516

MacMillan, D. S. 2006, in: International VLBI Service for Geodesy and Astrometry 2006 General Meeting Proceedings, Eds. D. Behrend and K. D. Baver, NASA/CP-2006-214140, p. 274.

Ojha, R., Fey, A. L., Johnston, K. J., Jauncey, D. L., Reynolds, J. E., Tzioumis, A. K., Quick, J. F. H., Nicolson, G. D., Ellingsen, S. P., Doodson, R. G., \& McCulloch, P. M. 2004, AJ 127,3609

Ojha, R., Fey, A. L., Charlot, P., Jauncey, D. L., Johnston, K. J., Reynolds, J. E., Tzioumis, A. K., Quick, J. F. H., Nicolson, G. D., Ellingsen, S. P., McCulloch, P. M., \& Koyama, Y. 2005, AJ 130, 2529 